\title{
Eine neue Kurve der Würm-Eiszeit
}

\author{
Von Paul Woldstedt, Bonn
}

Mit $1 \mathrm{Abb}$. im Text

$\mathrm{S} \mathrm{u} \mathrm{mmar} \mathrm{r}$. A new curve of the Last Glaciation in Middle Europe is presented. It is more complicated than the earlier ones. The Early Würm was humid and cool; the corresponding loess is the Younger Loess I. Where the ice-margin stood at the maximum of the Early Würm, we don't know. In the rather long-lasting Göttweig Interstadial we had a remarkable recession of the icefront. According to C14-measurements, the end of this interstadial may have occurred at about 28000 years B.C. The Middle Würm shows two advances with the Younger Loesses IIa and II'b, which are separated by the Paudorf Interstadial (end of this interstadial about 23000 years B.C.). The maximum of the Würmian ice-advance occurred about 16000-18000 years B.C., when the climate was extremely cold and dry.

Im Jahre 1954 habe ich den ersten Versuch gemacht, den Ablauf der Würm-Eiszeit in einer Kurve schematisch darzustellen. Eine verbesserte Kurve gab ich 1956. Heute lege ich einen dritten Versuch (Abb. 1) vor in der Hoffnung, dem wahren Verlauf der WürmEiszeit immer näher zu kommen $\left.{ }^{1}\right)$. Jede neue Kurve ist etwas komplizierter als die vorhergehende.

Vorstoß und Rückzug des eiszeitlichen Klimas gingen nicht in einem Zuge vor sich, sondern in zahlreichen Schwankungen offenbar recht verschiedenen Ausmaßes. Schwankungen dieser Art wurden von Helga REICH (1953) in den Schieferkohlen des bayerischen Alpenvorlandes nachgewiesen. Hier kommt man für den frühen Abschnitt der WürmEiszeit bereits zu Schneegrenzdepressionen, die bei $600-1000 \mathrm{~m}$ liegen. Schwankungen charakterisieren auch die mittleren Teile der Würm-Eiszeit - hier sind sie besonders durch die Lösse registriert - und, wie wir seit langem wissen, ebenso den letzten Teil, wo außer der Alleröd-Schwankung zum mindesten noch die Bölling-Schwankung vorhanden ist.
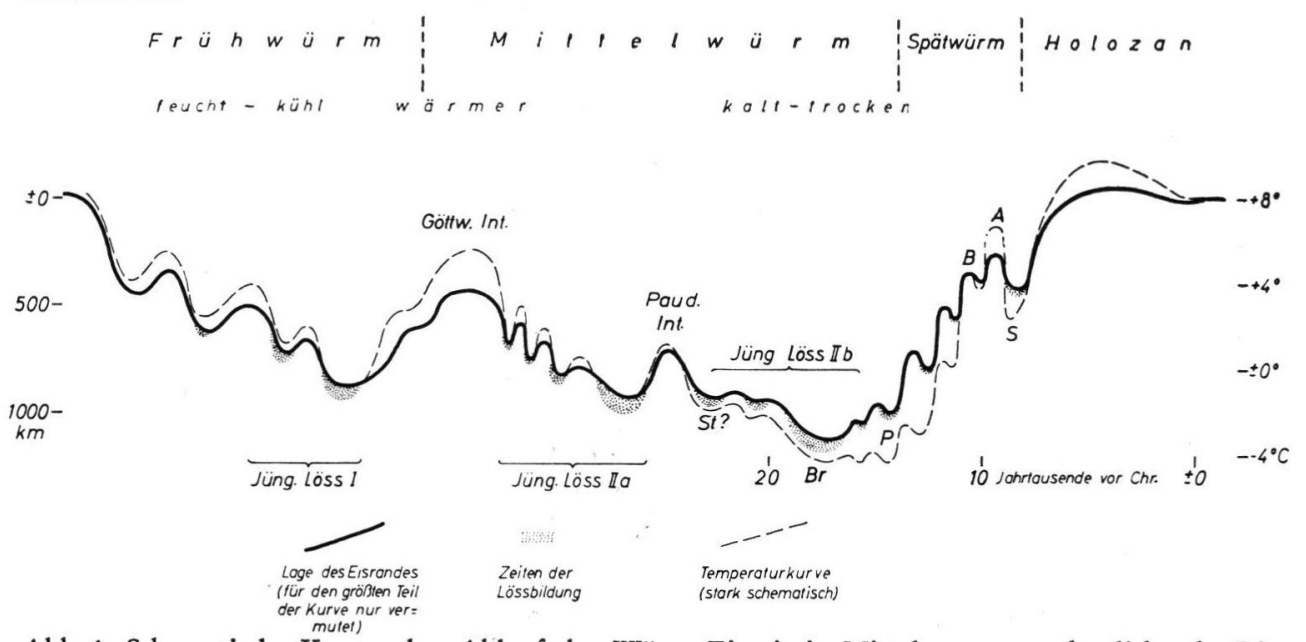

Abb. 1. Schematische Kurve, den Ablauf der Würm-Eiszeit in Mitteleuropa verdeutlichend. Die kräftig ausgezogene Kurve gibt die Lage des Eisrandes der nordischen Vergletscherung, in Kilometern vom (theoretischen) Eiszentrum aus gemessen (vgl. linke Skala), die gestrichelte Kurve die (roh geschätzten) Jahresmittel (rechte Skala) an. Eine Zeitskala ist nur bis $20000 \mathrm{~J}$. v. Chr. angegeben. St? $=$ Stettiner, $\mathrm{Br}=$ Brandenburger, $\mathrm{P}=$ Pommersches, $\mathrm{S}=$ Salpausselkä-Stadium; $\mathrm{B}=$ Bölling-, $\mathrm{A}=$ Alleröd-Interstadial.

1) Diese Kurve wurde dem V. Inqua-Kongreß in Madrid (Sept. 1957) in einem Vortrage vorgelegt. 
Die Kurve des einsetzenden eiszeitlichen Klimas (bzw. der Temperaturen) ging unter Schwankungen bis zu einem ersten Minimum, das durch den unteren Jüngeren Löß ("Jüng. Löß I“) repräsentiert wird. In Frankreich ist dieser Jüngere Löß I öfters zweigeteilt. Die Temperaturen gingen in diesem Abschnitt offenbar noch nicht so tief herunter wie später. Dagegen scheint es verhältnismäßig feucht gewesen zu sein. Urgeschichtlich wird dieser ältere Abschnitt der Würm-Eiszeit, der als Fr ühw ü rm bezeichnet wird, in der Hauptsache durch Moustier-Industrien verschiedener Prägung charakterisiert.

Es folgte dann eine stärkere Erwärmung, in der sich die Göttweiger Verlehmung bildete. Wie hoch die Temperatur wieder anstieg, ist nicht genau zu sagen. Im südlichen Mitteleuropa kam es in einzelnen, den Refugien nahegelegenen Gebieten wohl schon zur Wiedereinwanderung einzelner Laubbäume. Im übrigen Mitteleuropa hatten wir wahrscheinlich eine gemäßigt-subarktische Waldsteppe, hauptsächlich mit Birke, Kiefer und Arve. Wie lange dieser Abschnitt des Göttweiger Interstadials dauerte, entzieht sich vorläufig unserer Kenntnis. In dem darauffolgenden Abschnitt herrschte zunächst offenbar ein mehrfacher rascher Klimawechsel. F. BRANDTNER $(1954,1956)$ hat auf das öftere Abwechseln von Löß- und Schwarzerdebildungen in diesem Abschnitt hingewiesen. Die Temperatur ging in Schwankungen weiter herunter, bis schließlich das Minimum der Würm-Eiszeit erreicht wurde. Das war offenbar erst gegen Ende dieses Abschnittes der Fall. Mittlerer und oberer Jüngerer Löß, d. h. das, was in der Literatur meist als "Jüngerer Löß II" und „Jüngerer Löß III" bezeichnet wird (in Abb. 1 JL IIª und JL II' genannt), gehören in diesen Abschnitt, wie auch das zwischen ihnen gelegene Paudorfer Interstadial.

Der geschilderte mittlere Abschnitt der Würm-Eiszeit wird am besten als M i t t e 1w ü r m bezeichnet, wobei das Göttweiger Interstadial mit eingerechnet wird. Urgeschichtlich ist es hauptsächlich durch das sich ausbreitende Jungpaläolithikum, d. h. durch Aurignac, Gravette, Solutré usw., charakterisiert.

Es folgt das $\mathrm{S}$ p ä $\mathrm{t}$ w ü r m, in dem die Temperaturen unter wahrscheinlich kräftigen Schwankungen allmählich wieder anstiegen, bis vor etwa 10000 Jahren die Nacheiszeit begann. Urgeschichtlich ist der Spätwürm-Abschnitt, der dem früheren „Spätglazial“ entspricht, hauptsächlich durch die Magdalen-Industrien charakterisiert.

Eine andere als die 1956 von mir vorgeschlagene Einteilung in Früh-, Mittel- und Spätwürm hat H. Gross (1956) vorgeschlagen. Er unterscheidet ein „Alt“- und ein „Hauptwürm“, die durch das Göttweiger Interstadial getrennt werden.

Es trifft zu, daß die Würm-Eiszeit durch das Göttweiger Interstadial zweigeteilt wird. Nennt man aber den älteren Abschnitt „Altwürm“, so müßte man logischerweise den jüngeren „Jungwürm“ nennen, wie es auch z. B. E. KRAus (1955) macht. Die Bezeichnung "Jungwürm" ist aber teilweise schon in ähnlicher Weise verwendet worden wie "Spätwürm“, so daß hier Verwechslungen auftreten könnten. Ich halte deshalb die Einteilung in Früh-, Mittel- und Spätwürm für besser. Sie entspricht den auch sonst in der Geologie üblichen Gepflogenheiten. Man könnte höchstens im Zweifel darüber sein, wohin man das Göttweiger Interstadial stellen will, d. h. ob in das Früh- oder das Mittelwürm. Ich persönlich halte es für richtiger, es in das Mittelwürm zu stellen. -

Es entsteht nun die Frage, wie die hauptsächlich aus den Erscheinungen des nicht vereisten Gebietes abgeleitete Klimakurve mit dem Gang der nordischen (und alpinen) Vereisung zu kombinieren ist. Das kann zunächst nur vermutungsweise geschehen. In Abb. 1 ist eine zweite Kurve (kräftig) dargestellt, die den mutmaßlichen Gang des Eisrandes der nordischen Vergletscherung wiedergibt. Es wird dabei angenommen, daß die Maximalausdehnung des nordischen Eises, d. h. das Brandenburg-Frankfurter Stadium, wahrscheinlich mit Einschluß des Pommerschen Stadiums, dem ober e n Jüngeren Löß (II b) entspricht. Wahrscheinlich gehört zu dieser peripheren Endmoränengruppe auch noch das 
überfahrene Stettiner Stadium (das wohl nicht in den Frühwürm-Abschnitt gehört, wie ich 1956 annahm). Im alpinen Gebiet entsprechen dieser norddeutschen Endmoränengruppe das Zürich-, Killwangen- und Schlieren-Stadium.

Der mittlere Jüngere Löß (II ${ }^{a}$ ) würde einer etwas älteren Vorstoßgruppe entsprechen, die von der maximalen durch das Paudorfer Interstadial getrennt war. Davor ist dann das Göttweiger Interstadial anzunehmen, in dem der Eisrand sicher weit zurückgegangen ist - wie weit, das entzieht sich für Norddeutschland völlig unserer Beurteilung. Für die Alpen ergeben sich vielleicht aus den Untersuchungen über die Salzofenhöhle (vgl. bes. E. ScHmid 1957) Anhaltspunkte. Der u n te re Jüngere Löß (I) schließlich würde den späteren Vorstößen des Frühwürm-Abschnittes entsprechen (deren genaue Zahl wir bisher nicht kennen). Welches Maximum in diesem Frühwürm-Abschnitt vom Eise erreicht worden ist, wissen wir weder aus dem nordischen noch aus dem alpinen Gebiet.

Zum Schluß ist zu prüfen, wie weit die Kurve durch exakte Altersbestimmungen unterbaut werden kann²). Das soll hier nur ganz kurz. geschehen, da eine ausführliche Behandlung dieser Frage in der ebenfalls in diesem Bande erscheinenden Arbeit von H. Gross vorgesehen ist.

Der Ausgang der Eem-Interglazialzeit konnte bisher noch nicht exakt erfaßt werden. Er liegt (wahrscheinlich erheblich) „früher als 51000 J. v. Chr." In ein Interstadial des Frühwürms gehört offenbar das von S. T. ANDERSEN (1957) untersuchte Vorkommen von Brörup-Hotel in Jütland. Die C ${ }^{14}$-Bestimmung durch T AubER \& DE VRIEs (in diesem Band) kommt zu einem Alter von „mehr als 50000 J. v. Chr.“. Die von Andersen angenommene Einstufung in das Göttweiger Interstadial ist recht unwahrscheinlich. Bei diesem haben wir allerdings sichere Daten erst für den mittleren und späteren Teil. Etwa in das Optimum des Göttweiger Interstadials muß die aus der Salzofenhöhle bei Bad Aussee (österr. Alpen) stammende Holzkohlenprobe mit etwa $32000 \mathrm{~J}$. v. Chr. ${ }^{3}$ ) gehören (DE VRIEs \& WATERbolk 1958). In einen späteren Teil des Göttweiger Interstadials gehört offenbar das Aurignac I (mit „Knochenspitzen mit gespaltener Basis“) der IstallosköHöhle in Ungarn, das HL. DE VRIEs (in diesem Band) zu etwa $27700 \mathrm{~J}$. v. Chr. bestimmte. Für die Aurignac-Kulturschicht Willendorf II/4 ergab sich nach demselben Autor ein Alter von rund $30000 \mathrm{~J}$. v. Chr. So können wir das Ende des Göttweiger Interstadials bei etwa 28-30000 J. v. Chr. annehmen. Für das Ende des Paudorfer Interstadials ergeben mehrere $\mathrm{C}^{14}$-Bestimmungen (Unter-Wisternitz und Pollau, $\mathrm{H}_{\mathrm{L}}$. DE Vries in diesem Band) ein Alter von rund $23000 \mathrm{~J}$. v. Chr. Die Maximalausdehnung des nordeuropäischen Würm-Eises kann vorläufig nur geschätzt werden (ca. 16000-18000 J. v. Chr.?). Auf die Spätwürm- und Nacheiszeit, für die dann zahlreiche Altersbestimmungen vorliegen, sei hier nicht weiter eingegangen. -

Temperatur- und Eisrandkurve verlaufen in Abb. 1 diskordant zueinander. Für den Frühwürm-Abschnitt ist die Temperaturkurve oberhalb der Eisrandkurve gezeichnet worden in der Annahme, daß hier die Feuchtigkeit recht bedeutend war und die Eisausdehnung durch Temperatur und Fe u chigke it bedingt war. Erst im MittelwürmAbschnitt steigt die Temperatur unter die Eisrandkurve herunter. Jetzt macht sich die große Eisansammlung bemerkbar und wirkt temperaturmindernd auf die Umgebung. Unter verhältnismäßig starken Schwankungen geht dann das ganze Phänomen zurück.

Wir können den Ablauf der Würm-Eiszeit vergleichen mit dem normalen Gang eines kräftigen Winters: Kühl und feucht im Spätherbst und Frühwinter; dabei Schwankungen der Temperatur, die nach einer ersten kälteren Zeit im Dezember zu einer relativ warmen Periode mit Blühen einzelner Bäume usw. führen können - das wäre das Göttweiger

2) Die hier geäußerten Ansichten weichen z. T. von denen von Hl. DE Vries ab.

3) Mit Absicht werden hier stark abgerundete Zahlen eingesetzt. 
Interstadial. Dann setzt nach Weihnachten der eigentliche Winter ein. Aber noch ist der Kältehöhepunkt nicht erreicht. Dieser tritt erst Anfang Februar ein, wo wir normalerweise auch die geringsten Niederschläge haben. Jetzt ist es $\mathrm{kalt} u \mathrm{nd} \mathrm{trocken}$. Es folgt der Übergang zum Frühling mit einzelnen stärkeren Kälterückfällen.

\section{$\mathrm{Sch}$ if ten - Nachweis}

Andersen, S. Th.: New investigations of Interglacial Fresh-Water Deposits in Jutland. E. u. G. 8, S. 181-186, 1957.

Brandtner, F.: Jungpleistozäner Löß und fossile Böden in Nieder-Ósterreich. - E. u. G. 4/5, S. 49 bis 82, 1954. - - Lößstratigraphie und paläolithische Kulturabfolge in Niederösterreich und den angrenzenden Gebieten. - E. u. G. 7, S. 127-175, 1956.

Gross, Hugo: Das Göttweiger Interstadial, ein zweiter Leithorizont der letzten Vereisung. E. u. G. 7, S. 87-101, 1956.

Kraus, E.: Zur Zweigliederung der südbayerischen Würmeiszeit durch eine Innerwürm-Verwitterungsperiode. - E. u. G. 6, S. 75-95, 1955.

Reich, Helga: Die Vegetations-Entwicklung der Interglaziale von Großweil-Ohlstadt und Pfefferbichl im bayrischen Alpenvorland. - Flora 140, 1953.

Scrmid, Elisabeth: Von den Sedimenten der Salzofenhöhle. - Sber. österr. Akad. Wiss., math.-nat. Kl., Abt. I, 166, S. 43-55, Wien 1957.

TAuber, H., \& DE VRIEs, Hl.: Radiocarbon measurements of Würm-interstadial samples from Jutland. - E. u. G. 9, S. 69-71, 1958.

DE VRIES, H1.: Radiocarbon Dates for upper Eem and Würm-interstadial samples. - E. u. G. 9, S. 10-17, 1958.

Woldstedt, Paul: Saaleeiszeit, Warthestadium und Weichseleiszeit in Norddeutschland. - E. u. G. 4/5, S. 34-48, 1954. - - Uber die Gliederung der Würm-Eiszeit und die Stellung der Lösse in ihr. - E. u. G. 7, S. 78-86, 1956.

Manuskr. eingeg. 25. 5. 1958.

Anschrift d. Verf.: Prof. Dr. Paul Woldstedt, Bonn, Argelanderstr. 118. 